\title{
Shunt freedom and clinical resolution of idiopathic intracranial hypertension after bariatric surgery in the pediatric population: report of 3 cases
}

\author{
Kimberly B. Hoang, MD, ${ }^{1}$ Kristopher G. Hooten, MD, ${ }^{1,2}$ and Carrie R. Muh, MD1 \\ ${ }^{1}$ Department of Neurosurgery, Duke University, Durham, North Carolina; and 2Department of Neurosurgery, University of Florida, \\ Gainesville, Florida \\ Idiopathic intracranial hypertension $(\mathrm{IIH})$, formerly known as pseudotumor cerebri, is a disease of elevated intracranial \\ pressure that is thought to develop due to impaired CSF absorption related to elevated venous sinus pressure in the \\ setting of increased intraabdominal and thoracic pressures. Symptoms can be disabling and, if left untreated, can lead to \\ permanent visual loss. Previous treatments directed toward vision preservation include CSF diversion through shunting \\ and optic nerve sheath fenestration. Recently, attention has been turned toward surgical weight loss strategies as an \\ alternative to shunt treatment. The authors present a report of 3 patients with adolescent-onset IIH that was treated at \\ the authors' institution (Duke University) in whom bariatric surgery was pursued successfully. The patients had previously \\ undergone CSF shunting at ages 12,15, and 23 years. They were shunt dependent for a collective average of 3.3 years \\ prior to bariatriwc surgery. All patients reported "low-pressure" or postural headaches after bariatric surgery that correlated \\ with dramatic reduction in their weight. Two of the 3 patients had their shunts removed and continued to be shunt free 1.5 \\ years later at last follow-up; the third patient remained shunt dependent with the pressure set at $200 \mathrm{~mm} \mathrm{H}_{2} \mathrm{O}$. Given the \\ significant complications inherent to multiple shunt revisions, earlier intervention for weight loss, including bariatric surgery, \\ in these patients might have prevented complications and the associated health care burden. The authors recommend a \\ multidisciplinary approach for IIH treatment with early consideration for weight loss interventions in select patients. \\ https://thejns.org/doi/abs/10.3171/2017.6.PEDS17145
}

KEY WORDS idiopathic intracranial hypertension; pseudotumor cerebri; weight loss surgery; bariatric surgery; gastric bypass; shunt; hydrocephalus

I DIOPATHIC intracranial hypertension (IIH), formerly known as pseudotumor cerebri, is a disease of elevated intracranial pressure (ICP) in the absence of any underlying identifiable cause. Patients most commonly present with headache, but symptoms can include visual disturbance, tinnitus, nausea, vomiting, diplopia, and, rarely, focal neurological deficit. ${ }^{3,13} \mathrm{IIH}$ most commonly affects obese females of childbearing age. In young children $(<$ 12 years) there is no definite association with obesity and a higher incidence of underlying secondary causes. ${ }^{8,28} \mathrm{In}$ older children and adolescents (12-15 years), however, the number of reports in the published literature regarding an association between IIH and obesity is increasing. These patients have risk factors that are similar to those in the adult population. With the growing obesity epidemic expanding into the pediatric and adolescent populations, there is also an increasing incidence of IIH..$^{9,14,28}$ As a result, pediatric neurosurgeons will likely be facing more referrals for the treatment of IIH in the future.
Current etiology hypotheses suggest that the increase in ICP seen with IIH may be partly due to raised intrathoracic and intraabdominal pressures, which are commonly seen in obese patients.,31 In addition to weight reduction and medical therapies, surgical treatment options are typically reserved for vision preservation and include CSF diversion via shunting and optic nerve sheath fenestration. Over the past 2 decades, there has been an increasing incidence in the use of bariatric weight reduction surgery for the treatment of IIH in the adult population. ${ }^{15}$ However, there is a paucity of articles describing weight reduction surgery in the pediatric population, especially in regard to IIH. We present a series of 3 patients who developed IIH as adolescents and initially underwent shunt treatment, followed by bariatric surgical treatment at a later time, with great success. These patients are now shunt free or essentially shunt free with improvement in symptoms that directly correlate with their weight reduction. Interestingly, all patients experienced symptoms of overdrainage headaches follow- 
TABLE 1. Case series summary

\begin{tabular}{ccccccccc}
\hline $\begin{array}{c}\text { Case } \\
\text { No. }\end{array}$ & Sex & $\begin{array}{c}\text { Weight Loss } \\
\text { Method }\end{array}$ & $\begin{array}{c}\text { No. of } \\
\text { ED Visits }\end{array}$ & $\begin{array}{c}\text { Shunt } \\
\text { Type }\end{array}$ & $\begin{array}{c}\text { No. of } \\
\text { Shunt-Related Ops }\end{array}$ & $\begin{array}{c}\text { Other IIH-Related } \\
\text { Ops }^{*}\end{array}$ & $\begin{array}{c}\text { Shunt } \\
\text { Freedom }\end{array}$ & $\begin{array}{c}\text { Notable Shunt-Related } \\
\text { Complications }\end{array}$ \\
\hline 1 & $F$ & Surgical & 13 & VP & 4 & & $\begin{array}{c}\text { VP shunt } \\
\text { at 200 } \\
\text { mm } \mathrm{H}_{2} \mathrm{O}\end{array}$ & $\begin{array}{c}\text { Prolonged externalization } \\
\text { for infection, multiple } \\
\text { malfunctions }\end{array}$ \\
\hline 2 & F & Surgical & 4 & VP, LP & 13 & $\begin{array}{c}\text { Bilat subtemporal decompression, } \\
\text { separate prolonged ICP monitoring }\end{array}$ & Yes & Multiple malfunctions \\
\hline 3 & F & Surgical & $>15$ & VP, VA & 4 & & Yes & Multiple malfunctions \\
\hline
\end{tabular}

ED = emergency department; $V A$ = ventriculoatrial.

* Does not include lumbar puncture

ing dramatic weight loss. Before committing children and teenagers to possible long-term implanted hardware for CSF diversion, the utility of weight loss surgery should be explored. We now advocate for early consideration of weight reduction surgery as a means to avoid the many unnecessary headaches and complications associated with CSF diversion.

\section{Case Reports}

In our series, patients were able to achieve symptom resolution with increasing shunt settings or shunt freedom through significant weight loss after bariatric surgery. In Table 1 we attempted to summarize the shunt-related points of contact these patients had with the health care system, although the encounters are likely incomplete because of the use of multiple hospital systems and practitioners outside the Duke University system. Preoperative findings on imaging in all of these patients were unremarkable and without evidence of cerebral sinus thrombosis.

\section{Case 1}

This female patient presented at 12 years of age with chronic headaches and restricted visual fields on confrontational ophthalmological examination without papilledema. She ultimately required ventriculoperitoneal (VP) shunt placement after multiple lumbar punctures revealed pressures greater than $50 \mathrm{~cm} \mathrm{H}_{2} \mathrm{O}$ and Diamox treatment failure. The patient underwent 2 shunt revisions and had 13 emergency department visits because of unresolved symptoms and side effects due to high doses of acetazolamide and furosemide. Eventually, she required shunt externalization for wound breakdown, which was complicated by appendicitis requiring appendectomy. Given the number of hospital visits and complications associated with her care, the patient elected to undergo gastric sleeve surgery 5 years after her initial shunt placement to treat her IIH (body mass index [BMI] 53 [180th percentile]). At the most recent follow-up visit (7 months after bariatric surgery) she had lost $60 \mathrm{lbs}$ (BMI 41 [140th percentile]). Her VP shunt has been set to $200 \mathrm{~mm} \mathrm{H}_{2} \mathrm{O}$, with the intent of eventually removing the shunt. Distal catheter breakage was recently found in the patient's abdomen, but, given the success of her shunt setting wean, she elected to avoid further shunt revision surgery and reported improvement in headaches and stability of symptoms at last follow-up (Fig. 1).
Case 2

This female patient presented at 15 years of age with symptoms of headache, back pain, urinary retention, double vision, and 1+ bilateral papilledema. She eventually underwent lumboperitoneal (LP) shunt placement, followed 1 year later by VP shunt placement. The patient subsequently underwent 8 revision surgeries, 5 lumbar punctures, and shunt flow studies that revealed unremarkable findings, thus requiring shunt access without any further intervention. Given her continued symptoms despite the aforementioned management and with the return of papilledema, the patient also underwent surgery for ICP monitoring with documented ICP greater than $30 \mathrm{~cm}$ $\mathrm{H}_{2} \mathrm{O}$. She subsequently underwent bilateral subtemporal decompressions. The following year, despite improvement in papilledema, with the presence of intractable headaches and working CSF diversion, the patient underwent ICP monitoring, which demonstrated ICPs less than $13 \mathrm{~cm}$ $\mathrm{H}_{2} \mathrm{O}$. Notably, during this period of treatment via lumbar puncture and shunting, her BMI trended up from 36 (120th percentile) to 46 (150th percentile) at the maximum. At 19 years of age, 4 years after her initial diagnosis, the patient underwent gastric bypass surgery. Over the course of 2 years, her BMI decreased from 46 to 31 (95th percentile). Her LP and VP shunts were set to higher settings and were successfully removed 8 and 10 months, respectively, after her bariatric surgery. She experienced resolution of her headaches and was able to return to light-duty work. She remains shunt free and symptom free at last follow-up, 1.5 years after shunt removal (Fig. 2).

\section{Case 3}

This female patient presented at 16 years of age with polycystic ovarian syndrome, obesity, and headaches. She was found to have elevated opening pressures on lumbar puncture and was initially treated medically with attempted weight loss and acetazolamide. Multiple lumbar punctures, failed medical therapy, severe daily intractable headaches, and persistent elevated ICP led to the placement of a VP shunt when she was 23 years old. After 4 shunt revisions including conversion to a ventriculoatrial shunt for persistent abdominal pain and catheter migration out of the peritoneum, the patient elected to undergo gastric sleeve surgery 14 months after her initial shunt placement. Although she did have complications related to her 


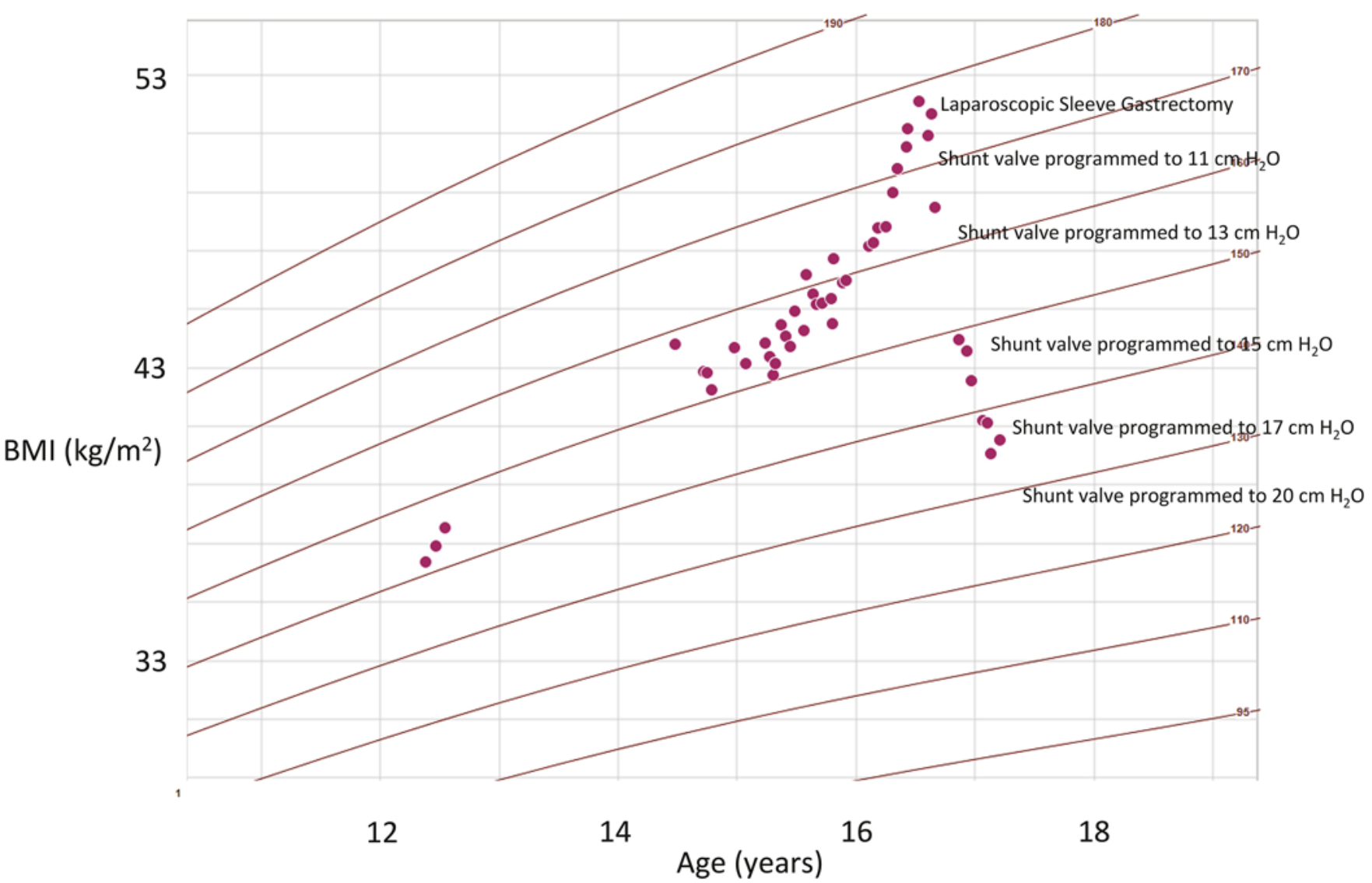

FIG. 1. Case 1. Graphic representation of BMI by age. Points represent clinic visits. Depicted is the process of increasing the shunt valve setting because of overdrainage symptoms, which correlated with dramatic weight loss. Figure is available in color online only.

gastric sleeve surgery that necessitated reoperation, which led to a gastric bypass, she was successfully weaned from her ventriculoatrial shunt, and it was removed over the course of 2 years. Her BMI decreased from 49.8 before bariatric surgery to 21.3 at the time of shunt removal. She remains shunt free 1.5 years later.

\section{Discussion}

IIH is a disease defined by clinically symptomatic elevation of ICP in the setting of normal anatomy and no apparent underlying cause. The estimated annual incidence of ICP in the pediatric population ranges from 0.47 to 1.2 per 1000,000 in developed countries, although there is no precise incidence estimate in the United States. ${ }^{11,33}$ In older children and adolescents there is an increasing incidence, particularly in obese adolescent females. ${ }^{28}$ If left untreated, the long-term sequela of elevated ICP is vision loss due to optic nerve damage. Surgical treatment options of CSF diversion and optic nerve sheath fenestration are aimed at preserving vision. All patients in our series underwent neurological and neurosurgical clinical evaluations, and all had multiple documented accounts of lumbar punctures revealing elevated ICP. The decision to place a VP or LP shunt was made in the presence of chronic headaches and/or papilledema/visual deficits after failure to control symptoms with conservative and medical therapy.
Although not completely understood, the pathophysiology of IIH is thought to result from impaired CSF absorption in the setting of elevated venous sinus pressure with increased intraabdominal and intrathoracic pressures associated with obesity., ${ }^{4,1931}$ While only 2 case reports currently exist in the pediatric literature,, 22 numerous studies in adults have shown improvement in symptoms and ICP through weight loss. ${ }^{18,21,26}$ In patients with CSF shunts, there is a documented risk of overdrainage headaches after weight loss.$^{29}$ Interestingly, all 3 of our patients demonstrated symptoms of low-pressure headaches correlating directly with weight loss. Our patients' headaches were defined by headache symptoms that improved with recumbency and worsened when upright. One theory is that this worsening is due to a reduction in their intraabdominal pressure and thus lower resistance to CSF diversion. Thus, we advocate for the use of programmable valves in this patient population, as the programmable shunt valves allow the shunt to be adjusted to higher pressures as patients continue to lose weight (Figs. 1 and 2). We monitored neurological symptoms after gastric surgery with interval outpatient follow-up every 3 months. No invasive testing to confirm ICP was done, but improvement in symptoms after reprogramming the shunt to higher pressures confirmed diagnosis in addition to providing therapy.

CSF shunting is obviously not without cost or risk. It is estimated that $20 \%-25 \%$ of all patients requiring a 


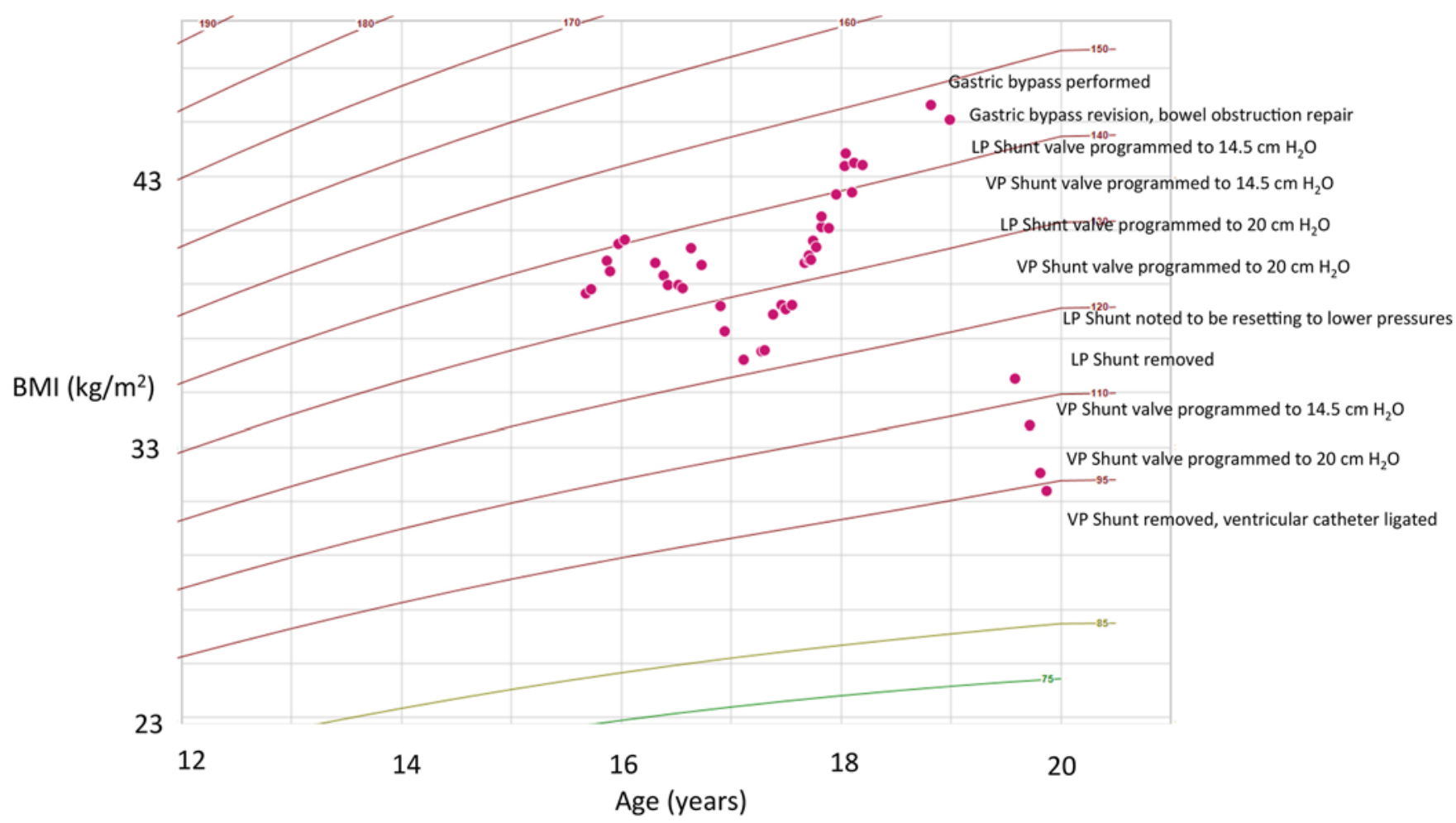

FIG. 2. Case 2. Graphic representation of BMI by age. Points represent clinic visits. Depicted is the process of increasing the shunt valve setting and shunt removals because of overdrainage symptoms, which correlated with dramatic weight loss. Figure is available in color online only.

shunt will have at least 1 complication. The complications related to shunt placement and revision in a population undergoing multiple revisions in a relatively short period of time are well demonstrated in this series. The published literature echoes this; in a large cohort of patients undergoing shunt surgery, the incidence of infection was $6.1 \%$, and the revision rate was $22.0 \%$. ${ }^{25}$ When patients with IIH are evaluated, shunt revision rates tend to be higher than those of other types of hydrocephalus and have been reported to be as high as $60 \%-100 \%$ for VP shunts and even higher for LP shunts., ${ }^{1,24,27}$ In our series of patients who underwent shunting, the revision rate was $100 \%$ with all patients requiring multiple revisions within 1 year. With the many complications requiring frequent evaluations, shunts in this population can place the patient at significant risk. Specifically, particular considerations regarding the details of shunt removal should be noted. Prior to removal in our series, the shunts were set at the highest pressure settings in consultation with a headache specialist. These patients underwent removal of the distal catheter and shunt valve. Because of the risk of hemorrhage and small ventricles associated with this disease, the ventricular catheter was tied off and sutured to the periosteum. If symptoms of ICP ever returned, the CSF diversion system could be easily reimplanted. After shunt removal, these patients were monitored overnight in the hospital, followed by outpatient follow-up for recurrence of symptoms related to elevated ICP and assessment for immediate postoperative complications. Although we did not leave a reservoir, in hindsight this may be a prudent technical strategy as the effects of bariatric surgery might be transient or not always effective, and ICP evaluation via a reservoir may be significantly easier than through a lumbar puncture in this population.

Furthermore, the economic and resource burden of the IIH population to the health care system should not go unmentioned. The median cost for shunt revision for failure is approximately $\$ 5000$, plus an additional estimated $\$ 40,000$ if the shunt becomes infected..$^{12,30}$ In addition, the average number of emergency department visits requiring some combination of neurosurgical consultation, lumbar puncture, shunt flow study/shuntogram, and shunt reprogramming was approximately 10 per patient, which is a substantial additional cost to the patient and health care system.

In our series we noted an immediate improvement in IIH symptoms after bariatric surgery, which directly correlated with dramatic weight loss as noted on the BMI charts. As a result of initial successes such as those reported here, our institution now offers weight loss surgery to adolescents. To qualify, patients must be at least 14 years old, have a BMI > 35 with at least one "serious" obesityrelated comorbidity or a BMI $>40$ with any obesity-related comorbidity. "Serious" comorbidities include Type 2 diabetes, severe obstructive sleep apnea, pseudotumor cerebri, and documented steatohepatitis. After referral to our weight loss center, patients undergo a 6-month trial of healthy lifestyle and conservative weight loss strategies and clinical and psychological evaluation before they are allowed to undergo any bariatric operation. 


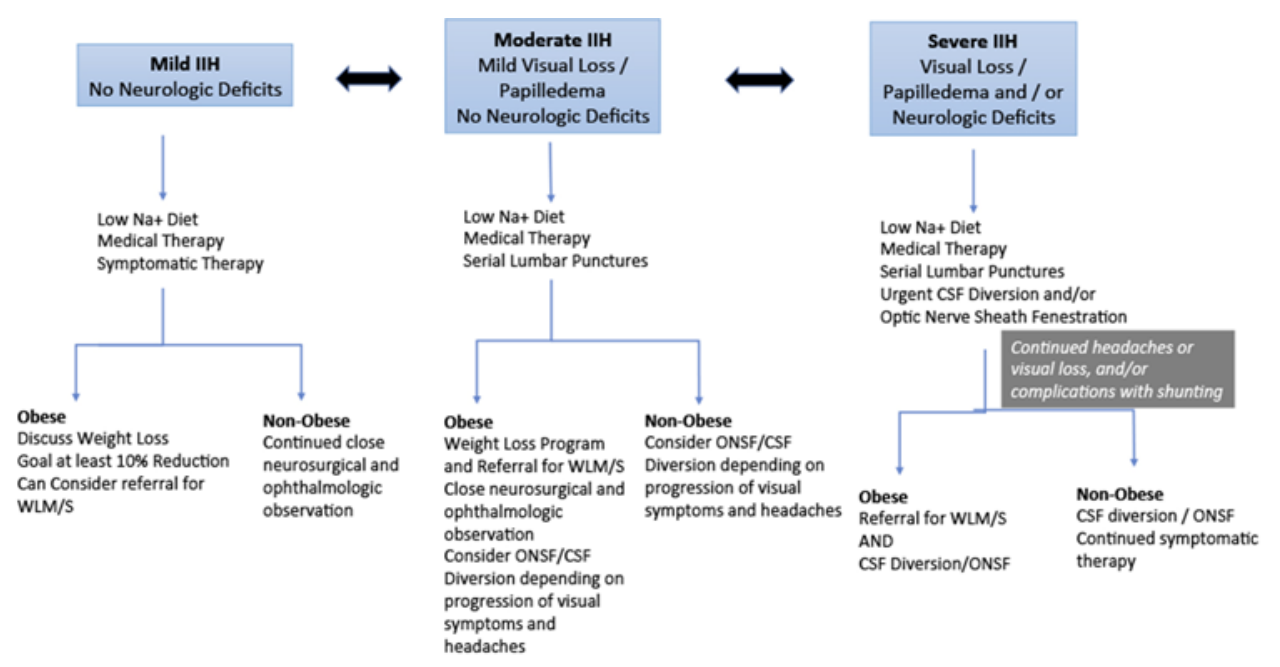

FIG. 3. Treatment algorithm for IIH patients. For mild or moderate IIH with obesity, we now recommend referral for weight management and weight loss surgery before permanent neurosurgical intervention. For nonobese patients we adhere to traditional treatment algorithms once secondary causes have been screened. ONSF = optic nerve sheath fenestration; WLM/S = weight loss management/surgery. Figure is available in color online only.

In general, bariatric surgery has been felt to be low risk and the most clinically effective and cost-effective treatment of obesity-related comorbidities in adults. ${ }^{17,20,32}$ The most common side effects include abdominal pain and changes in bowel habits, including constipation. With regard to adolescents compared with adults, pediatric patients differ biologically, psychologically, and socially. First, pediatric patients may have potential linear growth, and some surgical techniques may result in malabsorption and vitamin deficiencies important in a growing population..$^{5}$ Pediatric patients also have a higher incidence of eating disorders, depression, and anxiety, which are associated with greater noncompliance after surgery. ${ }^{10,16}$ Lastly, bariatric surgery performed in an adolescent patient raises the question of informed consent for operations that have life-altering results. ${ }^{6}$

Despite these differences, bariatric surgery has been demonstrated to improve weight loss, health, and psychosocial outcomes in adolescents. Because of the increase in positive results, we present a new algorithm that involves early referral to a multidisciplinary weight reduction team. For IIH, we present an algorithm for the management of patients, both obese and nonobese (Fig. 3). For obese patients, unless severe IIH with focal neurological findings or vision loss is present, we recommend referral to weight loss specialists before permanent neurosurgical intervention. All 3 patients at initial presentation would have met criteria for mild to moderate IIH and would have likely benefited from earlier referral to weight loss specialists for bariatric surgery consideration. For nonobese patients, once secondary causes have been evaluated, traditional treatment such as CSF diversion or optic nerve sheath fenestrations are considered.

As weight loss surgery continues to expand to younger populations, the use of bariatric surgery and effective nonsurgical weight loss strategies should be part of the neurosurgeon's armamentarium for patients with IIH. Currently, there is a paucity of long-term follow-up data, limited to 2 case reports, ${ }^{7,22}$ but there is an abundance of literature in the adult population that has supported the use of bariatric surgery as a treatment modality for $\mathrm{IIH}^{9,23}$ As with our small series, gastric bypass successfully helped resolve IIH symptoms (headaches and vision loss) in the other 2 case reports in the pediatric literature. With increasing obesity rates, we envision a multidisciplinary approach to this patient population. With the aid of general surgeons, weight loss physicians, psychologists, psychiatrists, nutritionists, neurologists, and ophthalmologists, IIH patients would ideally be referred to and reviewed by a multidisciplinary team to streamline care and avoid unnecessary procedures. Long-term results of this treatment should be followed prospectively with registries such as the Intracranial Hypertension Registry. ${ }^{2}$

\section{Conclusions}

$\mathrm{IIH}$ is increasing in the pediatric population. Pediatric neurosurgeons must be aware of the various treatment paradigms. We demonstrate the success of bariatric surgery in the adolescent population and advocate for early referral to weight loss physicians. The traditional treatment algorithm with all roads leading to CSF diversion surgery must be revisited.

\section{References}

1. Abubaker K, Ali Z, Raza K, Bolger C, Rawluk D, O’Brien D: Idiopathic intracranial hypertension: lumboperitoneal shunts versus ventriculoperitoneal shunts-case series and literature review. Br J Neurosurg 25:94-99, 2011

2. Aylward SC, Waslo CS, Au JN, Tanne E: Manifestations of pediatric intracranial hypertension from the intracranial hypertension registry. Pediatr Neurol 61:76-82, 2016

3. Ball AK, Clarke CE: Idiopathic intracranial hypertension. Lancet Neurol 5:433-442, 2006

4. Bloomfield GL, Ridings PC, Blocher CR, Marmarou A, Sugerman HJ: A proposed relationship between increased 
intra-abdominal, intrathoracic, and intracranial pressure. Crit Care Med 25:496-503, 1997

5. Browne AF, Inge T: How young for bariatric surgery in children? Semin Pediatr Surg 18:176-185, 2009

6. Caniano DA: Ethical issues in pediatric bariatric surgery. Semin Pediatr Surg 18:186-192, 2009

7. Chandra V, Dutta S, Albanese CT, Shepard E, Farrales-Nguyen S, Morton J: Clinical resolution of severely symptomatic pseudotumor cerebri after gastric bypass in an adolescent. Surg Obes Relat Dis 3:198-200, 2007

8. Cinciripini GS, Donahue S, Borchert MS: Idiopathic intracranial hypertension in prepubertal pediatric patients: characteristics, treatment, and outcome. Am J Ophthalmol 127:178-182, 1999

9. Curry WT Jr, Butler WE, Barker FG II: Rapidly rising incidence of cerebrospinal fluid shunting procedures for idiopathic intracranial hypertension in the United States, 1988-2002. Neurosurgery 57:97-108, 2005

10. Dawes AJ, Maggard-Gibbons M, Maher AR, Booth MJ, Miake-Lye I, Beroes JM, et al: Mental health conditions among patients seeking and undergoing bariatric surgery: a metaanalysis. JAMA 315:150-163, 2016

11. Dessardo NS, Dessardo S, Sasso A, Sarunić AV, Dezulović MS: Pediatric idiopathic intracranial hypertension: clinical and demographic features. Coll Antropol 34 (Suppl 2):217221,2010

12. Edwards NC, Engelhart L, Casamento EM, McGirt MJ: Cost-consequence analysis of antibiotic-impregnated shunts and external ventricular drains in hydrocephalus. J Neurosurg 122:139-147, 2015

13. Friedman DI, Jacobson DM: Idiopathic intracranial hypertension. J Neuroophthalmol 24:138-145, 2004

14. Gordon K: Pediatric pseudotumor cerebri: descriptive epidemiology. Can J Neurol Sci 24:219-221, 1997

15. Handley JD, Baruah BP, Williams DM, Horner M, Barry J, Stephens JW: Bariatric surgery as a treatment for idiopathic intracranial hypertension: a systematic review. Surg Obes Relat Dis 11:1396-1403, 2015

16. Herget S, Rudolph A, Hilbert A, Blüher S: Psychosocial status and mental health in adolescents before and after bariatric surgery: a systematic literature review. Obes Facts 7:233-245, 2014

17. Ibrahim AM, Ghaferi AA, Thumma JR, Dimick JB: Variation in outcomes at bariatric surgery centers of excellence. JAMA Surg 152:629-636, 2017

18. Johnson LN, Krohel GB, Madsen RW, March GA Jr: The role of weight loss and acetazolamide in the treatment of idiopathic intracranial hypertension (pseudotumor cerebri). Ophthalmology 105:2313-2317, 1998

19. Karahalios DG, Rekate HL, Khayata MH, Apostolides PJ: Elevated intracranial venous pressure as a universal mechanism in pseudotumor cerebri of varying etiologies. Neurology 46:198-202, 1996

20. Kaul A, Sharma J: Impact of bariatric surgery on comorbidities. Surg Clin North Am 91:1295-1312, ix, 2011

21. Kupersmith MJ, Gamell L, Turbin R, Peck V, Spiegel P, Wall $\mathrm{M}$ : Effects of weight loss on the course of idiopathic intracranial hypertension in women. Neurology 50:1094-1098, 1998

22. Leslie DB, Kellogg TA, Boutelle KN, Barnett SJ, Schwarzenberg SJ, Harrison AR, et al: Preserved vision without growth retardation after laparoscopic Roux-en-Y gastric bypass in a morbidly obese child with pseudotumor cerebri: 36-month follow-up. J Pediatr Surg 43:e27-e30, 2008

23. Manfield JH, Yu KK, Efthimiou E, Darzi A, Athanasiou T, Ashrafian H: Bariatric surgery or non-surgical weight loss for idiopathic intracranial hypertension? A systematic review and comparison of meta-analyses. Obes Surg 27:513-521, 2017

24. Menger RP, Connor DE Jr, Thakur JD, Sonig A, Smith E, Guthikonda B, et al: A comparison of lumboperitoneal and ventriculoperitoneal shunting for idiopathic intracranial hypertension: an analysis of economic impact and complications using the Nationwide Inpatient Sample. Neurosurg Focus 37(4):E4, 2014

25. Merkler AE, Ch'ang J, Parker WE, Murthy SB, Kamel H: The rate of complications after ventriculoperitoneal shunt surgery. World Neurosurg 98:654-658, 2017

26. Newborg B: Pseudotumor cerebri treated by rice reduction diet. Arch Intern Med 133:802-807, 1974

27. Niotakis G, Grigoratos D, Chandler C, Morrison D, Lim M: CSF diversion in refractory idiopathic intracranial hypertension: single-centre experience and review of efficacy. Childs Nerv Syst 29:263-267, 2013

28. Rangwala LM, Liu GT: Pediatric idiopathic intracranial hypertension. Surv Ophthalmol 52:597-617, 2007

29. Roth J, Constantini S, Kesler A: Over-drainage and persistent shunt-dependency in patients with idiopathic intracranial hypertension treated with shunts and bariatric surgery. Surg Neurol Int 6 (Suppl 27):S655-S660, 2015

30. Shannon CN, Simon TD, Reed GT, Franklin FA, Kirby RS, Kilgore ML, et al: The economic impact of ventriculoperitoneal shunt failure. J Neurosurg Pediatr 8:593-599, 2011

31. Sugerman HJ, DeMaria EJ, Felton WL III, Nakatsuka M, Sismanis A: Increased intra-abdominal pressure and cardiac filling pressures in obesity-associated pseudotumor cerebri. Neurology 49:507-511, 1997

32. Terranova L, Busetto L, Vestri A, Zappa MA: Bariatric surgery: cost-effectiveness and budget impact. Obes Surg 22:646-653, 2012

33. Tibussek D, Distelmaier F, von Kries R, Mayatepek E: Pseudotumor cerebri in childhood and adolescence-results of a Germany-wide ESPED-survey. Klin Padiatr 225:81-85, 2013

\section{Disclosures}

The authors report no conflict of interest concerning the materials or methods used in this study or the findings specified in this paper.

\section{Author Contributions}

Conception and design: all authors. Acquisition of data: Hooten, Hoang. Analysis and interpretation of data: all authors. Drafting the article: Hooten, Hoang. Critically revising the article: all authors. Reviewed submitted version of manuscript: Hooten, Hoang. Approved the final version of the manuscript on behalf of all authors: Hooten. Administrative/technical/material support: Hoang. Study supervision: Muh.

\section{Correspondence}

Kristopher G. Hooten, Department of Neurosurgery, Duke University, 4544 Hospital South, Box 3807, Durham, NC 27710. email: kristopher.hooten@neurosurgery.ufl.edu. 